ENCYCLOPEDDE Encyclopédie berbère

BERBERE

$6 \mid 1989$

6 | Antilopes - Arzuges

\title{
Arnobe (Arnobius)
}

J.-L. Charlet

\section{OpenEdition}

Journals

Édition électronique

URL : http://journals.openedition.org/encyclopedieberbere/2593

DOI : 10.4000/encyclopedieberbere. 2593

ISSN : 2262-7197

\section{Éditeur}

Peeters Publishers

\section{Édition imprimée}

Date de publication : 1 janvier 1989

Pagination : 909-911

ISBN : 2-85744-324-2

ISSN : 1015-7344

\section{Référence électronique}

J.-L. Charlet, «Arnobe (Arnobius) », Encyclopédie berbère [En ligne], 6| 1989, document A274, mis en ligne le 01 décembre 2012, consulté le 12 octobre 2020. URL : http://journals.openedition.org/ encyclopedieberbere/2593; DOI : https://doi.org/10.4000/encyclopedieberbere.2593

Ce document a été généré automatiquement le 12 octobre 2020

(c) Tous droits réservés 


\title{
Arnobe (Arnobius)
}

\author{
J.-L. Charlet
}

\section{L'homme}

1 Sur Arnobe, dont le nom atteste peut-être une origine grecque, nous ne savons que ce qu'en dit saint Jérôme, peut-être d'après une préface ou une notice qui aurait été placée en tête de ses œuvres. Il enseignait avec succès la rhétorique à Sicca Veneria (El Kef), en Numidie proconsulaire (Tunisie), sous le règne de Dioclétien (284-305), et eut pour élève le futur écrivain Lactance; des songes l'auraient incité à se convertir au christianisme ; comme l'évêque ne voulait pas lui faire "partager une croyance qu'il avait toujours combattue " (ce qui ne signifie pas nécessairement qu'il ait été un ennemi farouche et virulent des chrétiens), il entreprit d'écrire contre les païens des livres encore largement répandus à l'époque de Jérôme; par ce gage de sa piété, il obtint de devenir chrétien (Jérôme, uir. ill. 79 et chron. ad 327).

2 Ces livres « contre les païens » correspondent aux sept libres Aduersus nationes qui nous ont été transmis par un manuscrit carolingien et un manuscrit du $\mathrm{XI}^{\mathrm{e}}-\mathrm{XII}^{\mathrm{e}}$ siècle, copie du premier. Arnobe déclare au livre 1 (13) qu'il y a environ 300 ans que le christianisme existe, et, au livre 2 (71), que Rome a 1050 ans ou un peu moins. Ces deux premiers livres ont donc été écrits vers 300 et même, si Arnobe suit bien le comput varronien, au plus tard en 297; son désir de conversion devrait se situer en 295 ou 296. La composition des sept livres a prit plusieurs années: le livre 4 (36) fait sans doute allusion au premier édit de 303 qui prescrivait la destruction des églises et des livres chrétiens. L'état du dernier livre laisse supposer que la rédaction de l'ouvrage a été interrompue par la mort de l'auteur.

\section{L'œuvre}

3 Arnobe est un apologiste de l'ancienne école. Le titre même de son ouvrage le rattache à la première œuvre apologétique de Tertullien, l'Ad nationes (197). En guise d'introduction générale, le premier livre réfute l'accusation selon laquelle de nombreux 
malheurs se sont abattus sur le monde depuis l'apparition du christianisme: Arnobe montre que bien des catastrophes sont antérieures à la venue du Christ sur terre. Le livre 2, sorte de traité sur l'âme, s'en prend à la doctrine des " hommes nouveaux » sur la nature de l'âme; Arnobe peut viser ici des gnostiques aussi bien que des néoplatoniciens, en particulier Porphyre. Les cinq derniers livres polémiquent contre la religion gréco-romaine et l'incohérence de ses rites: son anthropomorphisme, l'absurdité, voire l'inconvenance de ses mythes, son exégèse allégorique, ses cultes et ses liturgies. Ils constituent une source incomparable de renseignements pour l'histoire de la religion romaine; ils sont aussi révélateurs du paganisme mystique qui se développait à la fin du $\mathrm{III}^{\mathrm{e}}$ siècle.

Arnobe met en œuvre dans l'Aduersus nationes une culture très vaste, mais parfois de seconde main: il puise souvent dans des manuels doxographiques. Arnobe connait Platon et le néoplatonisme. Ses critiques contre la religion païenne se fondent généralement sur Varron et Cicéron (De natura deorum), mais aussi Lucrèce et peut-être Cornelius Labeo; on relève aussi chez lui des réminiscences de Virgile, voire d'Ovide. Parmi les écrivains chrétiens, Arnobe semble connaître le grec Clément d'Alexandrie (Protreptique) et ses prédécesseurs latins Tertullien, Minucius Felix et surtout Cyprien (Ad Demetrianum). Mais sa culture chrétienne apparaît relativement superficielle. Il ne cite pas la Bible et ne fonde pas sur elle son argumentation: est-ce seulement parce qu'il éviterait d'utiliser des armes que les païens n'admettraient pas? En tout cas, son christianisme n'est pas d'une orthodoxie irréprochable. Arnobe est vaguement monothéiste et se représente Dieu comme indifférent et impassible. Pour lui, l'âme n'est pas créée par Dieu, mais par un démiurge. Sa christologie a des relents modalistes et docètes. Certaines bizarreries de sa théologie ne sont pas sans rapport avec les doctrines hermétistes, qu'il a dû connaître avant de se convertir.

5 Sur le plan littéraire, cette œuvre difficile, au style travaillé, raffiné et même alambiqué, vaut par la richesse bigarrée de sa langue, et surtout par la force de son imagination et la vigueur de sa verve satirique. Chez Arnobe, la critique en reste rarement à des arguments rationnels, mais s'exprime par des tableaux bouffons, voire grotesques, d'une ironie mordante.

\section{Le « patriotisme africain » d'Arnobe}

6 Arnobe manifeste dans l'Aduersus nationes un attachement très profond à son pays natal. Il est fier des héros africains et rappelle qu'Hannibal le Carthaginois a fait trembler Rome $(2,73 ; 7,50)$. Ce patriotisme africain l'amène à condamner les conquêtes de Rome $(2,1)$. Non seulement il parle en étranger de la domination romaine $(7,40)$, mais il la compare à un torrent qui a submergé et écrasé toutes les nations $(1$, 5) ; à ses yeux, Rome est née pour la perte du genre humain $(7,51)$.

Très attaché à la terre d'Afrique, Arnobe fait parfois allusion à des réalités africaines : les ravages causés par la sécheresse et les sauterelles $(1,3)$; le contraste qui peut exister, la même année, entre l'aridité de la Gétulie et de la Mauritanie Tingitane, et les riches moissons des Maures et des Numides $(1,16)$. Dans la liste des dieux païens qu'il critique $(1,36)$, il cite, à côté d'Hercule, de Castor et des dieux syriens, des dieux maures: Tisianes et Bucures Mauri, expression pour nous énigmatique et souvent corrigée, depuis Saumaise, en Titanes et Bocchores Mauri (voir le commentaire de l'édition H. Le Bonniec ad locum, pp. 297-298). S'agit-il d'un culte africain des Titans, mal 
connu (Diodore 3, 57), et des rois maures divinisés ? Le nom de Bucures est-il à mettre en rapport avec le dieu Bonchor (nom punique considéré par certains comme une contraction de Bodmelqart), à la tête des dii Mauri ? D'une manière plus générale, on a pu écrire que bien des points qui font difficulté dans la conception qu'Arnobe se fait de la divinité s'expliquent par le contexte de l'hétérodoxie africaine à la fin $\mathrm{du}_{\mathrm{III}}^{\mathrm{e}}$ siècle (M. Jufresa, 1973). Arnobe donne des renseignements très précieux sur la situation religieuse de l'Afrique romaine et sur les formes locales qu'y prend la religiosité populaire (S. Fasce, 1980).

\section{BIBLIOGRAPHIE}

\section{Éditions}

MARCHESI G., Arnobii Aduersus nationes libri VII, Corp. Script, lat. parav. t. 62, Torino $1953^{2}$, à préférer à l'édition de A. Reifferscheid (CSEL 4, Vienne 1875). Nouvelle édition en cours de publication aux Belles-Lettres, par H. Le Bonniec : livre I, Paris, 1982. Traduction anglaise par G. E. Mc Cracken (Westminster-London, 1949) ; italienne par R. Laurenti (Torino, 1962).

Études générales

MONCEAUX P., Histoire littéraire de l'Afrique chrétienne, t. 3, Paris 1905 (réimpr. 1966), chap. Arnobe, pp. 241-286.

GABARROU Fr., Arnobe, son œuvre, Paris 1921.

colombo S., « Arnobio Afro e i suoi sette libri Adversus nationes », Didaskaleion 9, 1930, pp. 1-124.

RAPISARDA E., Arnobio, Catania 1946.

Sur la vie et la chronologie d'Arnobe

NICHOLSON O. P., « The Date of Arnobius Aduersus Gentes », Stud. Patr. 15 (1975), 1984, pp. 100-107.

DUVAL Y.-M., « Sur la biographie et les manuscrits d'Arnobe de Sicca : les informations de Jérôme, leur sens et leurs sources possibles ", Latomus, 45, 1986, pp. 69-99.

Sur la pensée d'Arnobe et ses sources

TULLIUS Fr., Die Quellen des Arnobius im 4. 5. und 6. Buch seiner Schrift Adversus Nationes, Diss. Berlin 1934.

SCHEIDWEILER F., « Arnobius und der Marcionitismus », ZNTW 45, 1955, pp. $42-67$.

MAZZA M., « Studi Arnobiani I : la dottrina dei viri novi nel secondo libro dell’ Adversus Nationes di Arnobio », Helikon, 3, 1963, pp. 111-169.

SIRNA Fr.-G., « Arnobio e l'eresia marcionita di Patrizo », VChr 18, 1964, pp. 37-50.

MC DONALD H. D., « The Doctrine of God in Arnobius Adversus Gentes », Stud. Patr. 9, 1966, pp. 75-81.

LE BONNIEC H., « Arnobe témoin et juge des cultes païens », BAGB 1974, pp. 201-222.

JUFRESA M., « La divinidad y lo divino en Arnobio », BIEH 7, 1, 1973, pp. 61-64. 
LE BONNIEC H., « Le témoignage d'Arnobe sur deux rites archaïques du mariage romain », REL 54, 1976 (1977), pp. 110-129.

GAREAU E., « Le fondement de la vraie religion d'après Arnobe », CEA 11, 1980, pp. 13-23. Fasce S., «Paganesimo africano in Arnobio », Vichiana 9, 1980, pp. 173-180.

MADDEN J.-D., « Jesus as Epicurus. Arnobus of Sicca's Borrowings from Lucretius », CCC 2, 1981, pp. 215-222.

LAURENTI R., « Il platonismo di Arnobio », Stud. Filos., 4, 1981, pp. 3-54.

GIGON O., « Arnobio. Cristianesimo e mondo romano », Mondo class.) cristianesimo, Roma 1982, pp. 87-100.

LE BONNIEC H., « Échos ovidiens dans l'Aduersus nationes d'Arnobe », Caesarodunum XVII bis, 1982, pp. 139-151.

LE BONNIEC H., «L'exploitation apologétique par Arnobe du De natura deorum de Cicéron », Caesarodunum XIX bis, 1984, pp. 89-101.

LAURENTI R., « Spunti di teologia arnobiana », Orpheus VI, 1985, pp. 270-303.

AMATA B., Problemi di antropologia Arnobiana, Scienze Rel. 64, Roma 1984.

AMATA B., « Destino finale dell'uomo nell'opera di Arnobio di Sicca », Morte e immortalità nella catechesi dei Padri del III-IV secolo, Roma 1985, pp. 47-62.

LE BONNIEC H., « Un témoignage d'Arnobe sur la cuisine du sacrifice romain », REL 63, 1985, pp. 183-192.

Langue et style

LORENZ T., De clausulis Arnobianis, Diss. Breslau 1910.

GABARROU Fr., Le latin d'Arnobe, Paris 1921.

HAGENDAHL H., La prose métrique d'Arnobe, Göteborg Högskolas Arsskrift, t. 42, Göteborgs 1936.

BERKOWITZ L., Index Arnobianus, Hildesheim Olms, 1967.

OPELT I., « Schimpfwörter bei Arnobius dem Älteren », WS N.F. 9, 1975, pp. 161-173.

Survie d'Arnobe

KRAFFT P., Beiträge zur Wirkungsgeschichte des älteren Arnobius, Klassich-Philologische Studien Heft 32, Wiesbaden 1966.

INDEX

Mots-clés : Antiquité, Biographie 